\title{
Ressourcen finden, Ressourcen fördern
}

VON MATTHIAS HARTKAMP UND FRIEDRICH MECKMANN

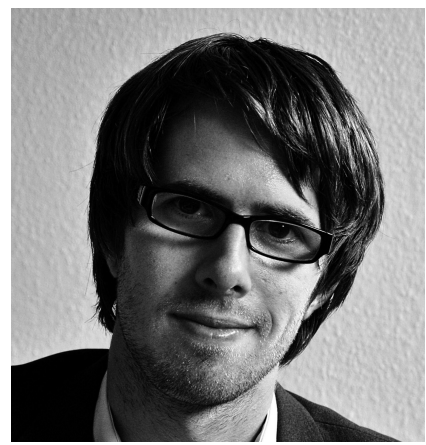

Matthias Hartkamp leitet die Stabstelle Projektmanagement bei der Benediktushof gGmbH in Reken. Das Unternehmen ist ein Rehabilitationszentrum für körper- und mehrfachbehinderte Menschen mit insgesamt 620 Plätzen in einem Berufsbildungswerk, in Wohnformen für Erwachsene, Kinder und Jugendliche sowie einer Werkstatt für behinderte Menschen.

matthias-hartkamp@t-online.de

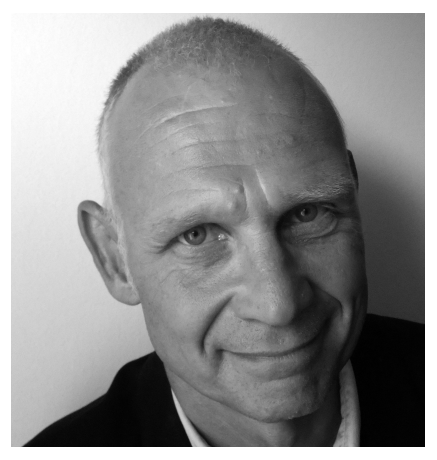

Friedrich Meckmann leitet den Fachdienst Sport und Rehabilitation im Benediktushof mit einem eigenen integrativen Sportverein. friedrichmeckmann@web.de

\author{
Immer mehr Unternehmen der Sozialwirtschaft \\ erkennen die Bedeutung der Gesundheit und \\ deren Einfluss auf das körperliche und seelische \\ Wohlbefinden sowie die Leistungsfähigkeit der \\ Mitarbeitenden. Ein eigenes Konzept für das \\ betriebliche Gesundheitsmanagement kann den \\ Beschäftigten, den Nutzern und dem Betrieb dienen.
}

Die Chancen der Einführung eines zeitgemäßen und bedarfsgerechten betrieblichen Gesundheitsmanagements liegen in der Verbesserung des Betriebsklimas, der Steigerung der Motivation sowie der Zufriedenheit der Mitarbeitenden. Gleichzeitig verhilft ein betriebliches Gesundheitsmanagement dazu, die Fehlzeiten und die damit einhergehenden Kosten für Unternehmen zu reduzieren. So gesehen darf Gesundheit nicht ausschließlich als Privatsache betrachtet werden, sondern sollte sich zu einem zentralen Zielwert für das Unternehmen entwickeln.

Als Folge des demografischen Wandels wächst branchenweit der Mangel an gut ausgebildeten Fachkräften. Gleichzeitig nehmen die Arbeitsverdichtung und die Anforderungen an die Mitarbeitenden stetig zu, was sich nicht zuletzt in der steigenden Anzahl von psychischen Erkrankungen bemerkbar macht. Vor diesem Hintergrund gewinnen die Personalbindung und Personalgewinnung sowie der Erhalt der Leistungsfähigkeit der Mitarbeitenden für Unternehmen zunehmend an strategischer Bedeutung.

Die Erhaltung der Leistungsfähigkeit umfasst sowohl die kognitiven als auch die psychischen und die körperlichen Ressourcen der beschäftigten Menschen. Durch gezielte Maßnahmen können diese erhalten bleiben, was wiederum dazu führt, dass die Mitarbeitenden länger im Unternehmen bleiben und somit einen Teil zur Bewältigung des Fachkräftemangels beitragen. Während die kognitive Leistungsfähigkeit durch zielgerichtete Fort- und Weiterbildungsmaßnahmen erhalten werden kann, beinhaltet das betriebliche Gesundheitsmanagement (betriebliches Gesundheitsmanagement) Maßnahmen zur Erhaltung der psychischen und physischen Arbeitsfähigkeit.

Zentraler Bestandteil des betrieblichen Gesundheitsmanagements ist eine gesundheitsorientierte Führungskultur, welche den Mitarbeiterinnen und Mitarbeitern ein Gefühl des Miteinanders, der Wertschätzung und der Anerkennung vermittelt.

\section{Gesundheit und Leistungsfähigkeit}

Anders als von der breiten Öffentlichkeit oftmals verstanden, umfasst der Begriff der Gesundheit mehr als das Fernbleiben von Krankheit. Vielmehr wird unter Gesundheit ein Zustand des vollständigen körperlichen, geistigen und sozialen Wohlergehens verstanden. (1)

Der salutogenetische Ansatz des amerikanischen Soziologen Aaron Antonovsky aus den 1970er Jahren stellt die Frage »Was erhält gesund? « in den Vordergrund. Bis dahin wurde die Gesundheit vom pathogenetischen Ansatz »Was macht krank? « betrachtet. Salutogenese und Pathogenese widersprechen sich hierbei nicht, nähern sich der Gesundheit aber von zwei unterschiedlichen Standpunkten. Für das betriebliche 
Gesundheitsmanagement stellt der Salutogeneseansatz, verbunden mit dem Kohärenzgefühl, eine wichtige Sichtweise dar, welche im weiteren Verlauf erneut aufgegriffen wird.

Unstrittig ist, dass die Ursachen mangelnder Gesundheit sowohl persönlicher wie auch arbeitsbedingter Natur sein können und ihren Ursprung auch in der fehlenden Vereinbarkeit von Arbeit und Privatleben haben können.

In der Vergangenheit stand bei Vorliegen einer Erkrankung die körperliche Verfassung des Patienten im Vordergrund, während der psychischen Konstitution oftmals nur ein untergeordneter Stellenwert zuteil wurde. Auch bedingt durch den starken Anstieg der psychischen Erkrankungen in den letzten Jahren kommt den psychischen Ressourcen eine immer größere Bedeutung zu.

Als Ursache der stetig ansteigenden psychischen Erkrankungen werden u.a. die technische Entwicklung (z. B. E-Mail, Smartphone) und die wachsende Arbeitsverdichtung (z. B. steigender Dokumentationsaufwand) angeführt. So gilt es, tagtäglich zahlreiche Entscheidungen zu treffen, kurzfristig und flexibel auf Veränderungen zu reagieren sowie die eigenen Kompetenzen optimal einzusetzen und auf dem neuesten Stand zu halten. Die steigende Zahl der psychischen Erkrankungen lässt sich auch an der steigenden Zahl von Tagen mit Arbeitsunfähigkeit mit der Diagnose "psychische Erkrankung « ablesen. Bei den Versicherten in der Krankenkasse DAK-Gesundheit sind diese beispielsweise zwischen 2006 und 2011 um rund 61 Prozent angestiegen (vgl. Abb. 1).

Mit Blick auf den Zusammenhang von Leistungsvermögen und Gesund- heit sind unternehmerische Erfolge und Misserfolge immer mehr als Gemeinschaftsleistungen $\mathrm{zu}$ betrachten und nicht primär an individuelle Mitarbeiterleistungen zu knüpfen. Vor diesem Hintergrund nehmen soziale Beziehungen zwischen den Kollegen, Teammitgliedern und Vorgesetzten eine bedeutsame Funktion ein. Sie fördern Motivation, Kreativität, Innovationsfähigkeit und tragen $\mathrm{zu}$ einem gesunden Betriebsklima bei.

\section{Leistungsfähigkeit erhalten}

Ein wichtiges Instrument zur Erhaltung der Leistungsfähigkeit einschließlich der psychischen Gesundheit stellt das betriebliche Gesundheitsmanagement dar. Damit dieses gezielt und passgenau greifen kann, bedarf es erstens der genauen Zielklärung auch zweitens der Bildung der notwendigen Strukturen einer intensiven Analyse der gesundheitlichen Situation im Unternehmen. Um einen Überblick über den Gesundheitszustand der Belegschaft zu erhalten, bieten sich für Führungskräfte und Personalverantwortliche eine Vielzahl von Analyseinstrumenten, wie beispielsweise Altersstrukturanalysen in Verbindung mit den Gesundheitsberichten der gesetzlichen Krankenkassen, der »Work-Ability-Index«, Arbeitsunfähigkeitsanalysen und Arbeitsplatzbegehungen an.

Der Work-Ability-Index (im Deutschen auch als Arbeitsfähigkeitsindex bekannt) ist ein standardisierter Fragebogen zur Ermittlung der individuellen gesundheitlichen Situation. Die Grundlage für diesen Index bildet das in Finnland entwickelte Konzept der Arbeitsfähigkeit. Demnach ist die Ar- beitsfähigkeit die Summe der Faktoren, die eine Person in einer bestimmten Arbeitssituation dazu befähigen, die ihr gestellten Arbeitsaufgaben erfolgreich zu bewältigen. (2)

Für das betriebliche Gesundheitsmanagement eignet sich dieses Instrument besonders, da es einfach zu handhaben und wissenschaftlich gut evaluiert ist. Im Gegensatz zu der vergangenheitsorientierten Betrachtung der Arbeitsunfähigkeitsdauer ermöglicht der Work-Ability-Index einen perspektivischen Blick in die Zukunft.

Ein weiteres Instrument, das vor dem Hintergrund des demografischen Wandels zunehmend an Bedeutung gewinnt, ist die Altersstrukturanalyse. Diese beschreibt die zahlenmäßige Verteilung der Belegschaft auf unterschiedliche Altersklassen. Aus dieser Auswertung lässt sich erkennen, ob in dem jeweiligen Unternehmen eine ausbalancierte, eine jugend- oder eine alterszentrierte Altersstruktur vorliegt.

Die Gesundheitsreporte der gesetzlichen Krankenkassen (3) belegen, dass es einen Zusammenhang zwischen dem Mitarbeiteralter sowie der Dauer und der Häufigkeit einer Arbeitsunfähigkeit gibt. Den Ausführungen der DAK-Gesundheit folgend, sind jüngere Personen häufiger krank als ihre älteren Mitmenschen. Gleichzeitig steigt aber die durchschnittliche Dauer der Arbeitsunfähigkeit mit dem Alter an. Für eine alterszentrierte Altersstruktur bedeutet dies, dass in Zukunft mit mehr langfristigen Krankheitsfällen zu rechnen ist. Gerade vor diesem Hintergrund gewinnt der präventive Ansatz des betrieblichen Gesundheitsmanagements bei alterszentrierten Belegschaften an Bedeutung.

\section{Wie ein betriebliches Gesundheitsmanagement aussehen kann}

Das betriebliche Gesundheitsmanagement verfolgt einen ganzheitlichen Ansatz und geht weit über die gesetzlichen Verpflichtungen zum Arbeitsschutz hinaus. In einem solchen betrieblichen Gesundheitsmanagement sind sowohl das betriebliche Eingliederungsmanagement als auch Maßnahmen der betrieblichen Gesundheitsförderung sowie umfangreiche Analysen und Auswertungen implementiert.
Abb. 1: Die steigende Zahl psychischer Erkrankungen macht sich auch in den Tagen mit Arbeitsunfähigkeit (AU) mit der Diagnose "psychische Erkrankung" bemerkbar, wie beispielsweise aus der DAK-Statistik hervorgeht. 


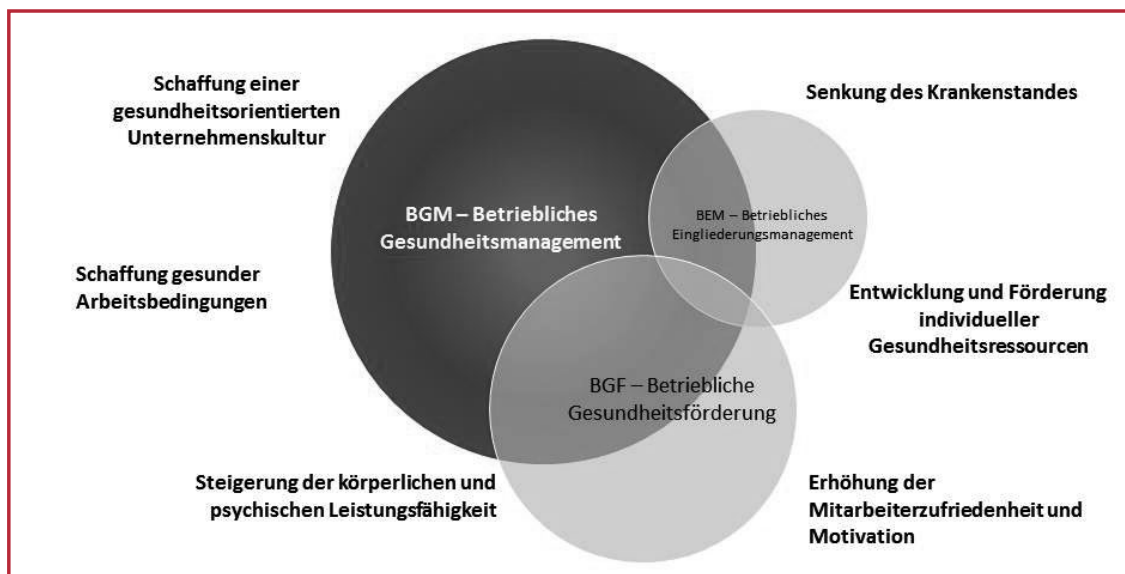

Abb. 2: Das Betriebliche Gesundheitsmanagement muss in ein Gesamtkonzept zum Thema Gesundheit eingebunden sein.

- Betriebliches Eingliederungsmanagement: Das gesetzlich vorgeschriebene betriebliche Eingliederungsmanagement dient der Wiederherstellung sowie der langfristigen Erhaltung und Förderung der Arbeitsfähigkeit von Mitarbeiterinnen und Mitarbeitern, die innerhalb von zwölf Monaten länger als sechs Wochen (42 Tage) ununterbrochen oder wiederholt arbeitsunfähig waren. (4) Dem Gesetzestext folgend ist ein Arbeitgeber verpflichtet, betroffenen Mitarbeitenden geeignete Hilfen anzubieten, um eine Arbeitsunfähigkeit zu überwinden und zukünftig vorzubeugen.

- Betriebliche Gesundheitsförderung: Im Jahr 1997 wurde von den Mitgliedern des Europäischen Netzwerkes für betriebliche Gesundheitsförderung die "Luxemburger Deklaration « verfasst, welche die Inhalte der betrieblichen Gesundheitsförderung definiert. Demnach umfasst diese alle Maßnahmen von Arbeitgebern, Arbeitnehmern und der Gesellschaft zur Verbesserung von Gesundheit und Wohlbefinden am Arbeitsplatz. Mittlerweile haben über 150 Unternehmen, Behörden sowie Gesundheits- und Sozialeinrichtungen diese unterzeichnet und sich somit den Prinzipien einer betrieblichen Gesundheitsförderung verpflichtet. (5)

- Betriebliches Gesundheitsmanagement: Ziel des betrieblichen Gesundheitsmanagements ist, die persönlichen Ressourcen von Mitarbeitenden zu stärken und sämtliche Aktivitäten zu deren Gesundheit nachhaltig in die betrieblichen Strukturen und Prozesse zu integrieren. Hierbei richtet sich das betriebliche Gesundheitsmanage- ment nicht vorrangig an den einzelnen Mitarbeitenden, sondern an das System Betrieb, das in den gesamten Gesundheitsförderungsprozess einbezogen wird. Voraussetzung dafür ist, dass das Thema Gesundheit in die Politik und Strategie des Unternehmens, zum Beispiel in Leitlinien, verankert ist (vgl. Abb. 2). Bei allen Aktivitäten sind alle Mitarbeitenden einzubeziehen - also auch alle Führungskräfte.

\section{Handlungsfelder und Praxisbeispiele}

Aus der Definition des betrieblichen Gesundheitsmanagements wird ersichtlich, dass es eine Vielzahl unterschiedlicher Ansatzpunkte und Instrumente beinhaltet. Die einzelnen Maßnahmen lassen sich unter unterschiedlichen Handlungsfeldern zusammenfassen, wie Arbeitsplatzgestaltung, Führung und Unternehmenskultur, Arbeitsorganisation, Bewegung und Stressabbau, Ernährung. Je nach Zielsetzung und unter Beachtung der gesundheitlichen Situation eignen sich unterschiedliche Instrumente. Wenn beispielsweise die psychischen Erkrankungen im Vordergrund stehen, kommt eine Rückenschule weniger infrage als eine Supervision oder eine Arbeitssituationsanalyse.

- Arbeitsplatzgestaltung: Mittels einer genauen Analyse der einzelnen Arbeitsschritte werden die an einem Arbeitsplatz anfallenden Aufgaben und Tätigkeiten auf ihre gesundheitlichen Auswirkungen überprüft. Ausgehend von diesen Ergebnissen gilt es, gesundheitsfördernde Maßnahmen abzuleiten, die über den bisherigen $\mathrm{Ar}$ - beitsschutz hinausgehen. Ein Instrument, mit dem die gesundheitsstörenden Faktoren ermittelt werden können, ist die Arbeitssituationsanalyse. Beispiel: Im Rahmen eines Projektes zum betrieblichen Gesundheitsmanagement in einer Kindertagesstätte wurde eine Arbeitssituationsanalyse durchgeführt. Als Ergebnis wurde das Heben der Kleinkinder auf den Wickeltisch als besonders anstrengend identifiziert. Die Erzieherinnen und Erzieher müssen die Kinder mehrmals am Tag auf diesen Tisch heben und anschließend wieder herunterlassen. Mit der Zeit führt dieses ständige Heben und Tragen zu erheblichen Rückenbeschwerden bei den Mitarbeitenden. Bei der Suche nach möglichen Lösungen wurde vorgeschlagen, an den Tisch eine Art Rutsche zu installieren, so dass die Kinder nach dem Wickeln den Tisch selbstständig verlassen können. Nach reichlicher Prüfung, insbesondere hinsichtlich der Sicherheit, konnte diese Idee verwirklicht werden. Heute verlassen die Kinder den Tisch rutschend, während gleichzeitig für das Personal ein körperlich anstrengender Arbeitsschritt entfallen ist.

- Führung und Unternehmenskultur: Der wichtigste, aber auch der langwierigste und oftmals aufwendigste Baustein im betrieblichen Gesundheitsmanagement zielt auf eine gesundheitsfördernde Unternehmensund Führungskultur ab. Um die Gesundheitsorientierung erfolgreich in die betriebliche Kultur zu implementieren, kommt den Führungskräften eine besondere Rolle zu, da diese zum einen als Vorbilder vorangehen müssen. Zum anderen aber auch, weil sie ihren Führungsstil um die Dimension "gesundheitsfördernde Führung « erweitern müssen. Dem SalutogeneseAnsatz folgend ist das sogenannte Kohärenz-Gefühl von zentraler Bedeutung für die Erhaltung der Gesundheit. Eine ausgeprägte Kohärenz, also ein hohes Maß an gesundheitlichen Ressourcen, liegt dann vor, wenn die Menschen den Sinn ihrer Tätigkeit erfassen, verstehen, warum sie was tun, und diese Handlungen ausführen können. Für das betriebliche Gesundheitsmanagement und das Handlungsfeld »Führung und Unternehmenskultur« hat dies zur Folge, 
dass die Führungskräfte ihren Mitarbeitenden die Sinnhaftigkeit ihrer Arbeit vermitteln müssen und sie dazu in die Lage versetzen, ihre Tätigkeiten ausführen und verstehen zu können. In speziellen Seminaren zu diesem Thema wird den Führungskräften gezeigt, wie ihr Führungsverhalten direkt und indirekt die Gesundheit, die Arbeitszufriedenheit sowie die Leistungsfähigkeit ihres Teams beeinflusst. (6) Eine weitere Möglichkeit, um die Gesundheitsorientierung in diesem Handlungsfeld zu verdeutlichen, ist die Fixierung der Gesundheit in betrieblichen Leitlinien, wie dem Leitbild oder den Führungsleitlinien.

- Arbeitsorganisation: Zu dem Handlungsfeld Arbeitsorganisation gehören die Maßnahmen, die nicht nur einen einzelnen Arbeitsplatz betreffen, sondern Auswirkungen auf die gesamte Organisation haben. Beispielhafte Interventionsmöglichkeiten in diesem Bereich stellen die Implementierung neuer Arbeitszeitmodelle sowie die Dienstplangestaltung dar. Durch die Verbesserung der Partizipationsmöglichkeiten der Mitarbeiterinnen und Mitarbeiter bei der Dienstplangestaltung kann die Teammotivation und somit auch die gesundheitlichen Ressourcen der einzelnen Teammitglieder stärken. Durch die Möglichkeit, sich selbst in die Dienstplangestaltung mit einzuschalten, kann sowohl die Motivation gefördert als auch Stress reduziert werden. Die Stressreduktion ergibt sich insbesondere dadurch, dass individuelle Lebenslagen und Situationen in der Dienstplangestaltung berücksichtigt werden. Dies kann bei- spielsweise durch ein »Wunschbuch « erfolgen, in das die Mitarbeitenden ihre bevorzugten Arbeitszeiten eintragen. Die Führungskraft, die für die Dienstplangestaltung zuständig ist, kann dann versuchen, diese Wünsche bei der Planung zu berücksichtigen - muss aber gleichzeitig auf eine Gleichbehandlung aller Kolleginnen und Kollegen achten.

- Bewegung und Stressabbau: Ziel dieses Handlungsfeldes ist nicht, dass alle Mitarbeitenden sportliche Höchstleistungen vollbringen. Vielmehr werden durch Sportangebote oder die Kooperation mit lokalen Sportvereinen und Fitnessstudios attraktive Anreize zum Sporttreiben geschaffen. So hilft ein moderates Walken beim Stressabbau und fördert nachweislich die Hirnaktivität. Zahlreiche Studien belegen, dass insbesondere ältere Menschen durch körperliche Aktivität ihr Gehirn strukturell verjüngen und dessen Leistungsfähigkeit steigern können. Eine Erhöhung der Konzentration verschiedener Botenstoffe (Neurotransmitter) im Gehirn wirkt sich zudem positiv auf Gedächtnisleistungen, das Lernvermögen und die emotionalen Prozesse aus. Durch die Bereitstellung von Schrittzählern, welche günstig zu haben sind, können Unternehmen ihre Mitarbeitenden zu regelmäßiger Bewegung anspornen: Schon 10.000 Schritte pro Tag haben nachweislich einen Nutzen für die Gesundheit.

- Ernährung: Eine ausgewogene Ernährung ist eine der Voraussetzungen, um die tägliche Arbeit erfolgreich zu meistern. Die Ernährung Ausdauertraining wie Joggen oder

\section{Checkliste-BGM}

$\checkmark$ BGM ist Bestandteil der Unternehmenspolitik

$\checkmark$ Strukturen und Verantwortlichkeiten für ein BGM sind vorhanden

$\checkmark$ Ausreichende personelle, materielle und finanzielle Mittel werden bereitgestellt

$\checkmark$ Alle Leitungskräfte werden zum Thema „Gesunde Führung“ regelmäßig geschult

$\checkmark$ Mitarbeiter können sich mit Ideen zur Gesundheitsförderung einbringen

$\checkmark$ Konkrete Maßnahmen zur Verbesserung der körperlichen und psychischen Gesundheit der Beschäftigten werden durchgeführt

$\checkmark$ Alle Mitarbeiter sind über die Möglichkeiten und Maßnahmen informiert

$\checkmark$ Gesundheitsrelevante Kennzahlen und Statistiken werden systematisch erfasst und ausgewertet

Abb. 3: Die Gesundheitsorientierung eines Unternehmens kann an zahlreichen Fakten abgelesen werden.

der Mitarbeitenden steht in einem unmittelbaren Zusammenhang mit der physischen und der kognitiven Leistungsfähigkeit. Die kurzfristigen Folgen einer ungesunden Ernährung zeigen sich beispielsweise durch Kopfschmerzen oder Leistungsabfall. Langfristig können Übergewicht und Herz-Kreislauf-Erkrankungen die Folge sein. Um eine gesunde Ernährung der Mitarbeitenden zu forcieren, bieten sich unterschiedliche Leistungen an. Durch das kostenfreie Bereitstellen von Mineralwasser kann ein Anreiz zum ausreichenden Trinken gesetzt werden. Sofern es eine Kantine gibt, kann sowohl durch die Preispolitik als auch durch die Speiseplangestaltung Einfluss auf das Ernährungsverhalten genommen werden.

\section{So kann es gehen: In fünf Schritten zum eigenen Gesundheitsmanagement}

ist ein Messinstrument zur Erfassung der Arbeitsfähigkeit von Erwerbstätigen. Er wird auch als Arbeitsfähigkeitsindex oder Arbeitsbewältigungsindex bezeichnet. Beim WAI handelt sich um einen Fragebogen, der entweder von den Befragten selbst oder von Dritten, z. B. Betriebsärzten bei der betriebsärztlichen Untersuchung, ausgefüllt wird. Ziel der Anwendung in Betrieben ist die Förderung bzw. Erhaltung der Arbeitsfähigkeit der Beschäftigten. Der WAI unterscheidet sich von klassischen Fragebogeninstrumenten in dem Sinne, dass es sich hierbei um ein sehr heterogenes Konstrukt handelt, welches sich durch zehn Fragen aus unterschiedlichen Bereichen zusammensetzt. Der WAI ist in diesem Zusammenhang kein Messinstrument im herkömmlichen Sinne, sondern in erster Linie als Index, Indikator oder als »Tool « zu verstehen, welches in engem Zusammenhang mit dem Konzept der Arbeitsfähigkeit anzuwenden ist. Der Fragebogen steht im Internet in einer Kurz- und in einer Langversion zum Herunterladen zur Verfügung. www.arbeitsfaehigkeit.uni-wuppertal.de/index.php?der-wai
Wichtige Voraussetzung für die erfolgreiche Implementierung eines ganzheitlichen betrieblichen Gesundheitsmanagements ist: Die Gesundheitsorientierung und die Gesundheitsförderung müssen sowohl in die strategische als auch in die operative Ebene der Unternehmensführung integriert werden. Gesundheit wird demnach zur »Chefsache« erklärt und als Führungsaufgabe verstanden. Neben einer systematischen Personalentwicklung und der Einführung von 
Strukturen und Prozessen des betrieblichen Gesundheitsmanagements geht es um die aktive Einbeziehung und Beteiligung aller Mitarbeitenden (vgl. Abb. 3).

\section{Zielplanung: Unter Berücksich-} - tigung der unternehmensspezifischen Rahmenbedingungen (Leistungsprogramm, Größe, Altersstruktur, Fehltage, Region etc.) zielt die strategische Zielplanung auf eine erste Standortbestimmung. Konkret geht es um die Frage, wo das Unternehmen steht - und warum es dort steht. Hierauf aufbauend werden übergeordnete Unternehmensziele in Bezug auf Gesundheit festgelegt und konkrete Projektmeilensteine definiert, derer es zur bedarfsgerechten und zielgerichteten Implementierung des betrieblichen Gesundheitsmanagements bedarf. Neben der Definition der Zielgruppe beinhaltet die Planung die zukünftigen Handlungsfelder, Interventionsziele sowie die Ressourcenplanungen (zeitlich, personell, monetär). Nach erfolgter Ressourcenplanung ist der nächste Schritt, die entsprechenden personellen Strukturen zu schaffen (beispielsweise mit der Gründung einer Projektgruppe).

2. Analyse: Bei der Analyse des Gesundheitszustandes des »Systems Unternehmen " geht es um die Erhebung und Analyse gesundheitsrelevanter Daten, unter Beachtung der Datenschutzvorschriften. Es empfiehlt sich, unterschiedliche Analyseinstrumente miteinander zu verknüpfen, um so ein differenziertes Bild zu erhalten. Neben den genannten Tools Work-Ability-Index und Altersstrukturanalyse können

Abb. 4.: Das betriebliche Gesundheitsmanagement kann in fünf Schritten aufgebaut werden.

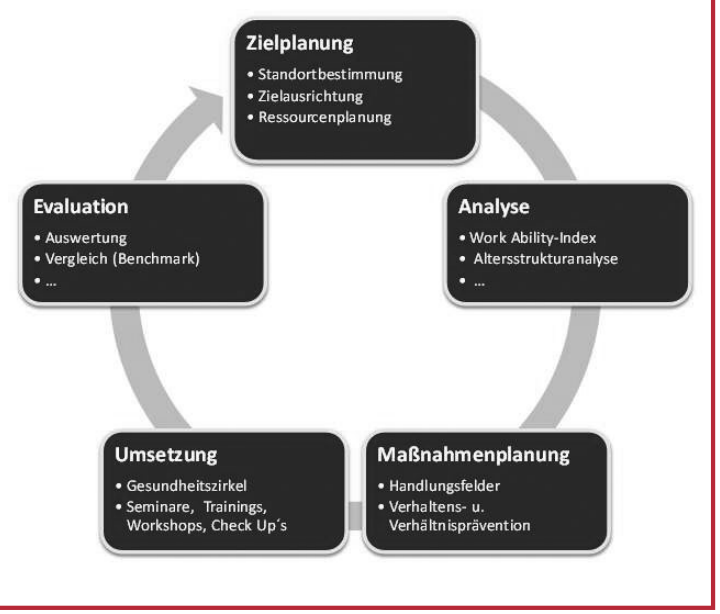

beispielsweise auch ArbeitsunfähigkeitsAuswertungen in Kooperation mit einer gesetzlichen Krankenkasse, Arbeitssituationsanalysen oder Arbeitsplatzbegehungen eingesetzt werden.

\section{3.} Maßnahmenplanung: Ausgehend von den Analyseergebnissen müssen passgenaue Maßnahmen entsprechend der Handlungsfelder entwickelt werden. Gemäß der Zielsetzung gilt es, die gesundheitlichen Ressourcen sowie das Wohlbefinden, die Motivation und die Leistungsfähigkeit der Mitarbeitenden zu fördern und zu erhalten. Sämtliche Maßnahmen sind auf die verschiedenen Ebenen bezogen werden: Individuum, Arbeitsbedingungen, Organisation, soziales Umfeld. Dies können neben den erwähnten Maßnahmen auch spezielle Workshops zu den Themen Bewegung, Ernährung, und Stressbewältigung sein oder auch medizinische Check-ups.

4. Umsetzung: Die Umsetzung der einzelnen Interventionen bezieht sich auf verhaltens- und verhältnisorientierte Maßnahmen. (7) Der vorab gebildete innerbetriebliche Gesundheitszirkel (als Projektgruppe) gewährleistet die Durchführung, Begleitung und Erfolgskontrolle der Maßnahmen. Insbesondere der Mitarbeiterpartizipation kommt in dieser Phase eine hohe Bedeutung zu.

5. Evaluation: Um die Erfolge des nagements bewerten zu können, gilt es, die durchgeführten Maßnahmen in regelmäßigen Abständen auf ihre Wirksamkeit hin zu überprüfen. Durch die Standardisierung der Datengrundlage kann eine Vergleichbarkeit geschaffen werden und so beispielsweise ein Benchmark mit anderen befreundeten Einrichtungen durchgeführt werden. Der Vergleich mit anderen Unternehmen der Branche ermöglicht wertvolle Rückschlüsse hinsichtlich der Interpretation gesundheitsspezifischer Entwicklungen und über deren Nutzen.

Das Zusammenspiel dieser fünf Aspekte ist entscheidend für das Gelingen eines betrieblichen Gesundheitsmanagements (vgl. Abb. 4).

\section{Fazit}

Das betriebliche Gesundheitsmanagement stellt einen wichtigen Baustein zur Bewältigung des demografischen Wandels in der Sozialwirtschaft dar.

Die im betrieblichen Gesundheitsmanagement getroffenen Maßnahmen verhelfen zum einen, die Arbeitsfähigkeit der Belegschaft langfristig zu erhalten und so krankheitsbedingte Neubesetzungen zu vermeiden. Anderseits können Mitarbeitende durch eine wertschätzende und gesundheitsorientierte Unternehmenskultur an das Unternehmen gebunden werden. Durch die Verknüpfung mit einer Personalmarketingstrategie kann das betriebliche Gesundheitsmanagement zudem mit dem Personalrecruiting verbunden werden.

Aber das betriebliche Gesundheitsmanagement rechnet sich für Unternehmen auch aus finanzieller Sicht. So haben unterschiedliche wissenschaftliche Studien die Wirtschaftlichkeit des betrieblichen Gesundheitsmanagements nachgewiesen. Diesen folgend liegt der Return on invest (ROI) des betrieblichen Gesundheitsmanagements zwischen $1: 2,3$ und 1:5,9.

\section{Anmerkungen}

(1) Vgl. die Verfassung der Weltgesundheitsorganisation: www.admin.ch/ ch/d/sr/i8/0.810.1.de.pdf.

(2) Vgl. Prümper, J.; Richenhagen, G. (2011).

(3) Vgl. hierzu beispielsweise DAK-Gesundheit: www.dak.de.

(4) Vgl. $\$ 84$ Absatz 2, SGB IX.

(5) Die "Luxemburger Deklaration « steht im Internet in deutscher Sprache zur Verfügung: www.netzwerkunternehmen-fuer-gesundheit.de/ luxemburger-deklaration.html.

(6) Vgl. hierzu beispielsweise das Seminarangebot »Gesundheitsfördernde Führung: Möglichkeiten und Grenzen« der Berufsgenossenschaft Gesundheitsdienst und Wohlfahrtspflege: https://www.bgw-online.de/ internet/generator/Inhalt/OnlineInhalt/Seminare/2012/Fuehrungskraefte/UMGF/UMGF.html?filter=freie.

(7) Während die Verhaltensprävention auf die Optimierung des menschlichen Verhaltens abzielt, beschäftigt sich die Verhältnisprävention mit der Gestaltung der Arbeitsbedingungen. 